\title{
Maternal near Miss: An Indicator of Maternal Health
}

\author{
Dr.Princey Rajakumari ${ }^{1}$, Dr.Manonmani ${ }^{2}$ \\ ${ }^{1}$ (Assistant professor, Department of OBG, Coimbatore Medical College Hospital, Coimbatore, India) \\ ${ }_{2}^{2}$ (Professor and HOD of Department of OBG, Coimbatore Medical College Hospital, Coimbatore, India)
}

\begin{abstract}
Maternal near miss is a newly arising concept with increasing popularity among maternal health care system. The purpose of this study is to find out the incidence and prognosis of near miss patients. This is a prospective observational study, conducted for 3 months period in a tertiary care centre. Near miss cases were identified according to operational guidelines published by Ministry of health and family welfare, Government of India in December 2014. This study will also compare the cause for maternal death occurred during the study period. Analysis of the data revealed that the incidence of near miss is 20 fold higher than the maternal death. Hypertensive disorder is the leading cause for near miss. Medical complications like heart disease carries higher mortality index than obstetric complications. Perinatal and neonatal mortality are significantly higher in near miss group when compared with general population. Maternal near miss is a potential indicator of maternal health. Notification and analysis of maternal near miss events will help in improving maternal health care system. It will also provide vital data for the policy makers to concentrate and strengthen the potential deficits so as to achieve safe mother hood.
\end{abstract}

Keywords - Maternal near miss, maternal death, severe maternal outcome, mortality index, perinatal mortality rate, neonatal mortality rate.

\section{Introduction}

Maternal death (MD) is only the visible portion of an iceberg. There are lot of patients who are coming with severe maternal morbid complication and getting well. This study is to determine the frequency of near miss events and its outcome among pregnant women over a period of 3 months in a tertiary care hospital in South India. This article also analyses the cause and frequency of maternal deaths to find out incidence and similarities with near miss events.

\section{Definition}

Near miss (NM) events are defined [1] as acute obstetric complication that threaten a women's life but not resulted in death because of medical care she received during pregnancy, labour or within 6 weeks after termination of pregnancy. Analysis of near miss events will reveal the cause for maternal morbidity and also the facility for treatment.

\section{Background}

Confidential enquires of maternal death is used to improve the care given to the mother so as to reduce the maternal mortality. The cause for death could be the delay in reporting to medical facility, may be because of social and cultural factors or inadequate transport facilities. There may be delay in referral from primary health care provider. There may be deficiency in treatment because of inadequate human resources and other facilities. The adequacy of referral services and treatment given in referral centre is also audited to reveal the cause for maternal deaths.

Near miss events are more frequent, than maternal death. It has several advantages as we can interview the patient herself to identify the pitfalls. Health care providers will also come out with more information as the patient has survived the complication.

\section{Hospital Settings}

This study is conducted at Coimbatore Medical College Hospital, Coimbatore, Tamilnadu. It is a State Govenment owned tertiary care teaching hospital in South India. High risk mothers are referred from primary health centres and Government head quarters hospitals. We receive patients from our own district and also from nearby districts namely Nilgiris, Erode and Tirupur which covers around 18,000sq.km. The district of Nilgiris is a hilly region where the transport facilities are scanty. The population covered is about 90 lakhs. All medical care given to the patient is provided free of cost. This centre provides emergency medical care over $24 \times 7$ days. Emergency surgeries, ICU facilities, blood and blood products transfusion, radiology services (CT, MRI.), haemodialysis, neonatal intensive care unit and other specialist services. Around 700 deliveries were conducted per month. 
The birth rate and death rate of the state of Tamilnadu is being 15.6, 7.3 respectively. The maternal death rate [5] is 90 per one lakh live births (MMR in India 178/100,000 live births in the year 2010-12). There is a steady decline in MMR throughout the state over the past few years. We are regularly conducting audit of all maternity deaths at district and state level to rectify the deficiency in the obstetric care. We also communicate to the referring centres regarding those patients for better management and early referral.

Now we focus on the patients who are about to die but survived by efficient management. This study also focuses on the cause for the near miss events and preventing factors. The study extends to analyse demography of the patient. A comprehensive analysis of near miss morbidity and maternal mortality among pregnant mother is done. This will also provide an insight about the quality of care given to the patient.

\section{Inclusion Criteria}

The World Health Organization defines a maternal near-miss case as "a woman who nearly died but survived a complication that occurred during pregnancy, childbirth or within 42 days of termination of pregnancy."

Severe maternal complications are defined as "potentially life-threatening conditions". This is an extensive category of clinical conditions, including diseases that can threaten a woman's life during pregnancy and labour and after termination of pregnancy.

Women with life-threatening conditions (WLTC) refers to all women who either qualified as maternal near-miss cases or those who died

In December 2014 the Ministry of Health \& Family Welfare Government of India Maternal Health Division published maternal near miss identifying criteria [2] and notification guidelines, so as to accelerate the decline of maternal deaths for achieving our national and international goals.

Criteria for identifying and notifying the MNM case have been put under three broad categories:

1). Pregnancy specific obstetric and medical disorders,

2). Pre-existing disorders aggravated during pregnancy,

3). Accidental / Incidental disorders in pregnancy.

Criteria (minimum three from each category) must be met with:

1). Clinical findings

2). Investigations

3). Interventions

Or any single criteria which signify cardio respiratory collapse.

Pregnancy specific obstetric and medical disorders include severe haemorrhage, Sepsis, Hypertension, Postpartum collapse, pregnancy related Liver dysfunction /failure and Cardiac dysfunction (e.g. Cardiomyopathy). Pre-existing disorders aggravated during pregnancy include Anaemia, Respiratory Dysfunctions, heart disease, Hepatic Dysfunction, Endocrinal disorders (e.g. Diabetic Ketoacidosis, Thyroid Crisis), Neurological and Renal Dysfunction.Accidental / Incidental disorders in pregnancy include accidental fall, burns, snake bite, poisoning, drug reactions, transfusion reaction, infection, embolism and infarction

\section{Materials And Methods}

It is a prospective observational study in patients who are admitted in our hospital during the months of July, August and September 2016 who fulfils the inclusion criteria are included in the study and followed up till discharge. Maternal death during the same period is also analysed and compared with near miss cases.

Data obtained is analysed with the following indicators

\subsection{Maternal Near-Miss Indicators}

A woman with life-threatening conditions (WLTC) refers to all women who either qualified as maternal near-miss cases or those who died. It is the sum of maternal near-miss and maternal deaths (WLTC = $\mathrm{MNM}+\mathrm{MD})$.

Severe maternal outcome ratio (SMOR) refers to the number of women with life-threatening conditions (MNM + MD) per 1000 live births (LB). This indicator gives an estimate of the amount of care and resources that would be needed in an area or facility $[\mathrm{SMOR}=(\mathrm{MNM}+\mathrm{MD}) / \mathrm{LB}]$.

MNM ratio (MNMR) refers to the number of maternal near-miss cases per 1000 live births (MNMR = MNM/LB). Similarly to the SMOR, this indicator gives an estimation of the amount of care and resources that would be needed in an area or facility.

Maternal near-miss mortality ratio (MNM: $1 \mathrm{MD}$ ) refers to the ratio between maternal near miss cases and maternal deaths. Higher ratios indicate better care.

Mortality index refers to the number of maternal deaths divided by the number of women with lifethreatening conditions expressed as a percentage $[\mathrm{MI}=\mathrm{MD} /(\mathrm{MNM}+\mathrm{MD})]$. The higher the index the more women with life-threatening conditions die (low quality of care), whereas the lower the index the fewer women with life-threatening conditions die (better quality of care). 
Perinatal outcome indicators (e.g. perinatal mortality, neonatal mortality or stillbirth rates) in the context of maternal near miss could be useful to complement the quality of care evaluation.

\section{Observation}

During the three months (July, August and September 2016), we had 3350 admission in labour ward. Total no of deliveries 1999, Total live births 1964.Total vaginal deliveries were 1121 and LSCS were 843.Total no of near miss cases identified was 283.Total no of maternal deaths were 14, including 1 brought dead patient and 1 patient died of oleander seed poisoning.

Of 283 near miss patients, $116(41 \%)$ women were primigravida. women presented in their $2^{\text {nd }}, 3^{\text {rd }}$ and $4^{\text {th }}$ pregnancy were $93(33 \%), 58(20 \%)$ and $16(6 \%)$ respectively. In maternal death group $33 \%(4)$ were primigravida, $33 \%$ were $2^{\text {nd }}$ and $33 \%$ were $3^{\text {rd }}$ gravida.

Table 1Parity

\begin{tabular}{|l|l|l|}
\hline Parity & NM (\%) & MD (\%) \\
\hline Primi & $116(41 \%)$ & $4(33 \%)$ \\
\hline 2 & $93(33 \%)$ & $4(33 \%)$ \\
\hline 3 & $58(20 \%)$ & $4(33 \%)$ \\
\hline$>=4$ & $16(6 \%)$ & - \\
\hline Total & 283 & 12 \\
\hline
\end{tabular}

More number of women in near miss group belongs to 21-30 yrs (75.3\%).similar trend is seen in maternal mortality group.

Table 2 Age of the mother

\begin{tabular}{|l|l|l|}
\hline Age of the mother & NM (\%) & MD (\%) \\
\hline$<20$ yrs & $38(13.4 \%)$ & $1(8.3 \%)$ \\
\hline $21-30$ & $213(75.3 \%)$ & $8(66.7 \%)$ \\
\hline $31-40$ & $30(10.6 \%)$ & $3(25 \%)$ \\
\hline$>40$ & $2(0.7 \%)$ & - \\
\hline
\end{tabular}

Analysis of Gestational age at the time of admission showed majority of near miss patients (33.6\%) were identified in term pregnancy; $32.2 \%$ were presented in 28-37 weeks. Those presented in $1^{\text {st }}$ and $2^{\text {nd }}$ trimester were $9.5 \%$ and $7.4 \%$ reectively. $17.3 \%$ were admitted postnatally. In maternal death group $41.7 \%$ were belong to $28-37$ weeks of gestation and $16.6 \%$ belong to term pregnancy. $41.7 \%$ patients admitted postnatally.

Table 3 Gestational age in weeks

\begin{tabular}{|l|l|l|}
\hline Gestational age(weeks) & NM (\%) & MD (\%) \\
\hline$<12$ & $27(9.5 \%)$ & - \\
\hline $12-28$ & $21(7.4 \%)$ & - \\
\hline $28-37$ & $91(32.2 \%)$ & $5(41.7 \%)$ \\
\hline$>37$ & $95(33.6 \%)$ & $2(16.6 \%)$ \\
\hline Post natal & $49(17.3 \%)$ & $5(41.7 \%)$ \\
\hline Total & 283 & 12 \\
\hline
\end{tabular}

The cause for maternal near miss in the study population is mainly due to hypertensive disorder contributing to $45.7 \%$. The other causes with decreasing frequency are haemorrhage (26.64\%), severe anaemia $(10.6 \%)$, neurological complications (10\%), infections $(5.6 \%)$, and heart disease $(4.6 \%)$.
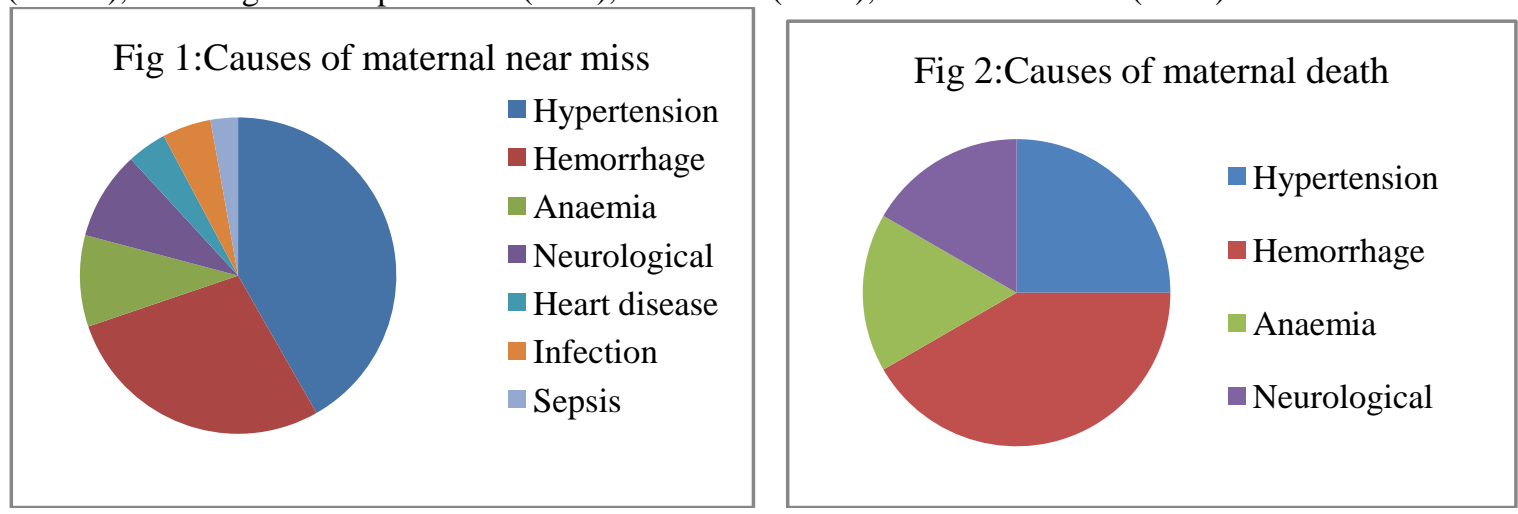

Among 283 near miss patients 134 patients had severe hypertensive disorder. 116 patients had severe preeclampsia and 18 patients had eclampsia. All patients were treated with injection magnesium sulphate, anti hypertensives and corticosteroids. Gestational age at the time of presentation was 28-37 wks in 62(46.3\%) patients, > $37 \mathrm{wks}$ in $48(35.8 \%)$ patients, $<28 \mathrm{wks}$ in $9(6.7 \%)$ patients and 15 patients referred postnatally. In patients with eclampsia, pregnancy was terminated within $24 \mathrm{hrs}$. In case of severe preeclampsia and imminent 
eclampsia termination delayed until corticosteroids completed if patient is stable. Vaginal delivery occurred in $62(46.3 \%)$ and caesarean delivery in $57(42.5 \%)$ of patients. Perinatal mortality of the patients with severe pre eclampsia and eclampsia were 168 per 1000 births, neonatal mortality being 38 per 1000 live births. It is significantly high when compared with perinatal mortality (30.51) and neonatal mortality (20.37) of the same period in our hospital. HELLP Syndrome was noted in 6 patients and 2 patients complicated with DIVC.

Haemorrhage was the next commonest cause for maternal near miss. It contributes to $26.64 \%$. Ante partum haemorrhage was occurred in 36 patients. Abruption placenta was diagnosed in 21 patients and 15 patients had placenta previa. Previous caesarean delivery was associated in $33 \%$ of patients with placenta previa. In placenta previa all patients delivered by caesarean section. In all patients bilateral uterine artery ligation was done before attempting placental removal. After placental delivery, placental bed suturing was done.5 patients needed step wise devascularisation to control bleeding. In spite of using uterotonics and all the above measures 4 patients needed hysterectomy in placenta previa.In patients with abruption 7 patients delivered vaginally, 10 patients needed caesarean delivery and 4 were admitted postnatally. 2 mothers died of APH during the same period, both of them had abruption Severe Post partum haemorrhage was found in 20 patients. 12 Patients referred postnatally following delivery.18 patients managed with medical methods and blood transfusion. 2 patients had undergone hysterectomy for uncontrolled bleeding. Exploration under anaesthesia was done in 1 patient with traumatic PPH. 2 patients died of atonic PPH during the study period. 19 patients presented with ruptured ectopic needed emergency laparotomy and blood transfusion. In all patients Laparotomy was done within $3 \mathrm{hrs}$ of admission. No maternal mortality was reported in hemorrhage in early pregnancy (e.g. abortion, ectopic, gestational Trophoblastic disease)

Sepsis was seen in 9 patients .4 of them presented following abortions before 20 weeks of gestation. All 4 had incomplete abortion for which manual vacuum aspiration done. Patient treated with higher antibiotics. Puerperal sepsis was found in 2 patients. 3 patients had post operative wound sepsis needed surgical drainage and higher antibiotics. In maternal death group a patient with severe preeclampsia and HELLP syndrome died of CVT following relaparotomy for suspected loculated infection.Sudden postpartum collapse and acute pregnancy related liver failure was not reported in the study period.

Among pre-existing disorder severe anaemia $(\mathrm{Hb}<7 \mathrm{grm} \%)$ is seen in 30 women, contributing to $11 \%$ of near miss cases. All patients were treated with packed cell transfusion, iron and vitamins supplementation. 2 of the patients also had severe thrombocytopenia needed platelet transfusion. Among the patients with anaemia 10 had vaginal delivery, 7 had caesarean section .3 of them had spontaneous abortion and 5 discharged antenatally .5 patients admitted postnatally for severe anaemia. Among maternal death one woman died of severe anaemia resulted in congestive cardiac failure.

Heart disease was found in 13(4.6\%) patients of whom 7 had rheumatic heart disease. 3 had congenital heart disease and 3 had cardio myopathy. Among heart disease 5 patients had vaginal delivery, 6 had caesarean section and 2 patients were admitted postnatally. All patients where admitted in ICU of them 4 Patients needed ionotropic support. In maternal mortality group 1patient had rheumatic heart disease. Postmortem pathology reports of another patient who was brought dead confirmed the diagnosis of cardiomyopathy.

Pregnancy in patient with cirrhosis and portal hypertension was reported in 1 patient. She delivered by Caesarean section. Diabetes mellitus presented with diabetic Ketoacidosis in 3 patients of near miss of which 2 patients presented in $3^{\text {rd }}$ trimester, 1 patient presented in $1^{\text {st }}$ trimester. All patients managed with insulin and intra venous fluid replacement. Spontaneous expulsion of dead foetus occurred in 1 patient, 1 had vaginal birth and 1 patient had caesarean section.

Neurological complications were present in 29 patients. Recurrent convulsions were seen in 13 patients with previous history of epilepsy. After ruling out the possibility of eclampsia all the patients were treated with anti epileptics. One patient needed complete paralysis with ventilatory support for seizure control. Cortical venous thrombosis was seen in 9 patients and intra cranial haemorrhage was seen in 5 patients. All the patients with intra cranial haemorrhage are treated with anti oedema measures, anti epileptics and hyper tension control. One patient underwent craniotomy and External ventricular drainage for Intra ventricular haemorrhage. That Patient was put on ventilatory support, higher antibiotics and antiepileptics. Tracheostomy was done as the patient needed prolonged ventilatory support. Patient recovered with minimal neurological deficit at the end of 6 weeks. 1 patient had increased intra cranial tension due to a road traffic accident delivered by caesarean section under general anaesthesia with the advice from neurosurgeon. 1 patient had extra pyramidal syndrome. Cerebro vascular accident was seen in 2 patients of maternal mortality group.

3 patients had Acute kidney injury due to obstetric complication needed haemodialysis in near miss group. All the patients had severe preeclampsia. 1 patient underwent haemodialysis in maternal mortality group.

Infection was seen in 16 patients. 3 of them had dengue, 4 were HIV positive, 5 were HbsAg positive and 4 had severe lower respiratory tract infection. None of the maternal mortality group die of infection.

2 patients had pulmonary embolism, confirmed by pulmonary angiogram in near miss group. 3 patients died of pulmonary embolism in mortality group. Amniotic fluid embolism was seen in 1 patient in 
maternal mortality group. Other rare causes of Near miss were pregnancy in patient with myasthenia gravis (1 patient), hypokalemic paralysis seen in 1 patient.

Pulmonary oedema was seen in 2 patients who were put on ventilator to maintain oxygenation in near miss cases. 3 patients in maternal mortality group had pulmonary oedema.

Table 4 Organ Dysfunction

\begin{tabular}{|l|l|l|}
\hline Organ Dysfunction & $\mathrm{NM}(\%)$ & $\mathrm{MD}(\%)$ \\
\hline Cardiac & $5(1.7 \%)$ & $9(64.3 \%)$ \\
\hline Respiratory & $11(3.8 \%)$ & $8(57.1 \%)$ \\
\hline Haematological & $17(6 \%)$ & $4(28.5 \%)$ \\
\hline Uterine & $17(6 \%)$ & $1(7.1 \%)$ \\
\hline Neurological & $13(4.5 \%)$ & $2(14.2 \%)$ \\
\hline Hepatic & $8(2.8 \%)$ & - \\
\hline Renal & $3(1.1 \%)$ & $1(7.1 \%)$ \\
\hline \hline
\end{tabular}

In Near miss cases haematological dysfunction like DIVC, HELLP Syndrome was seen in 17 patients, uterine dysfunction $(\mathrm{PPH})$ requiring devascularisation or hysterectomy was seen in 17 patients, neurological dysfunction like altered consciousness, persistent convulsions was seen in 13 patients. Respiratory dysfunction needing ventilator support was seen in 11 patients. Cardiac dysfunction requiring advanced cardio pulmonary resuscitation was given for 5 patients. Hepatic dysfunction was seen in 8 patients and renal dysfunction was seen in 3 patients .Organ dysfunction was more frequent in maternal mortality group. 9 suffered cardiac dysfunction, 8 had respiratory dysfunction, 4 had haematological dysfunction, 2 patients had neurological dysfunction. Renal and uterine dysfunction was seen in 1 patient each. Multi organ dysfunction (>=2organ system involvement) was seen more commonly in maternal mortality group $50 \%$ (7) than in near miss group $2.4 \%(7)$.

Table 5 Interventions done

\begin{tabular}{|l|l|l|}
\hline Intervention & NM & MD \\
\hline ICU admission & 208 & 12 \\
\hline CPR & 5 & 9 \\
\hline Ventilatory support & 11 & 8 \\
\hline Blood transfusion & 88 & 7 \\
\hline Higher antibiotics & 24 & 8 \\
\hline Haemodialysis & 3 & 1 \\
\hline Ketoacidosis management & 3 & - \\
\hline Laparotomy for ectopic & 19 & - \\
\hline $\begin{array}{l}\text { Laparotomy for } \\
\text { rupture uterus }\end{array}$ & 2 & - \\
\hline Caesarean hysterectomy & 10 & 1 \\
\hline Stepwise devascularisation & 6 & - \\
\hline Bladder injury repair & 1 & - \\
\hline $\begin{array}{l}\text { Exploration } \\
\text { under anaesthesia }\end{array}$ & 1 & 1 \\
\hline
\end{tabular}

In Near miss patients average duration of hospital stay is 13.66. In maternal mortality group admission to death interval is $<7$ days $1 \mathrm{n} 9$ patients in which 3 patients died within 2 hrs. 1 patient who stayed for 31 days , under gone relaparotomy for suspected loculated infection, died of CVT following surgery.2 patients were brought dead.

19 patients had laparotomy for ruptured ectopic, 8 had manual vacuum aspiration, laparotomy for rupture uterus in 2 patients. 49 patients were admitted postnatally and 18 patients discharged antenatally. Caesarean section rates are higher in maternal mortality group. LSCS rates in near miss group also more when compared with general population.

Table 6 Mode of delivery (MOD) and birth weight of babies in near miss patients

\begin{tabular}{|l|l|l|l|}
\hline & $\mathrm{NM}[\mathrm{n}(\%)]$ & $\mathrm{MD}[\mathrm{n}(\%)]$ & Others [n(\%)] \\
\hline $\begin{array}{l}\text { MOD } \\
\text { Vaginal birth }\end{array}$ & $93(48 \%)$ & $1(17 \%)$ & $1027(52 \%)$ \\
\hline LSCS & $102(52 \%)$ & $5(83 \%)$ & $736(42 \%)$ \\
\hline Birth wt(kg) & & & \\
\hline$<1$ & $19(9.74 \%)$ & - & $10(0.5 \%)$ \\
\hline $1-1.5$ & $26(13.3 \%)$ & - & $56(2.8 \%)$ \\
\hline $1.5-2$ & $34(17.4 \%)$ & $3(50 \%)$ & $126(6.3 \%)$ \\
\hline $2-2.5$ & $68(34.9 \%)$ & - & $383(19.2 \%)$ \\
\hline $2.5-3$ & $30(15.4 \%)$ & $3(50 \%)$ & $946(47.3 \%)$ \\
\hline$>3$ & $18(9.2 \%)$ & - & $478(23.9 \%)$ \\
\hline
\end{tabular}


Table 7 Perinatal outcome

\begin{tabular}{|l|l|l|l|l|}
\hline Perinatal outcome & \multicolumn{2}{|l|}{ NM } & \multicolumn{2}{l|}{ MD } \\
\hline & alive & dead & alive & dead \\
\hline At birth & 167 & 28 & 4 & 2 \\
\hline At 7 days & 158 & 9 & 4 & - \\
\hline
\end{tabular}

Perinatal mortality in babies of near miss mothers where 189.7 per 1000 births. In maternal mortality group the rate was 333.3 per 1000 births. In our hospital perinatal mortality is reported as 30.5 per 1000 births during the same period. Neonatal mortality is 53.89 and 500 per 1000 live births in near miss and maternal mortality group respectively. In our hospital neonatal mortality is 20.37 per 1000 live births.

Table 8 Apgar score (5 minute) of babies

\begin{tabular}{|l|l|l|}
\hline Apgar & NM & MD \\
\hline$>=7 / 10$ & 141 & 4 \\
\hline $4 / 10-6 / 10$ & 18 & - \\
\hline$<=3 / 10$ & 8 & - \\
\hline
\end{tabular}

\section{Data Analysis}

On analysing the data collected over 3 months period total no of women with life threatening complications(near miss + maternal death) is 297.SMO ratio (severe maternal outcome ratio)is 151.2 per 1000 live births. Maternal near miss ratio is 144.1 per 1000 live births. Maternal near miss mortality ratio 20.21. This says that the incidence of near miss is 20 times more frequent than maternal death incidence. Mortality index $[\mathrm{MD} /(\mathrm{MNM}+\mathrm{MD})]$ is 4.71.

There is no significant difference in age distribution, parity and gestational age at the time of admission between near miss group and maternal death group.

The cause for maternal near miss in the study population is mainly due to hypertensive disorder contributing to $45.7 \%$. The other causes with decreasing frequency are haemorrhage (26.64\%), severe anaemia $(10.6 \%)$, neurological complications $(10 \%)$, infections $(5.6 \%)$, and heart disease $(4.6 \%)$.

Table 9 Cause and mortality index (MI) of maternal near miss

\begin{tabular}{|l|l|l|l|}
\hline Cause & NM $(\%)$ & MD $(\%)$ & MI $(\%)$ \\
\hline Hypertension & $134(45.7 \%)$ & $3(21.4 \%)$ & 2.19 \\
\hline Hemorrhage & $90(32 \%)$ & $5(35.7 \%)$ & 5.3 \\
\hline Anaemia & $30(10.6 \%)$ & $2(14.2 \%)$ & 6.25 \\
\hline Neurological & $29(10 \%)$ & $2(14.2 \%)$ & 6.45 \\
\hline Heart disease & $13(4.6 \%)$ & $2(14.2 \%)$ & 13.33 \\
\hline Infection & $16(5.6 \%)$ & - & - \\
\hline Sepsis & $9(3 \%)$ & - & - \\
\hline
\end{tabular}

The leading cause for maternal death was haemorrhage $35.7 \%$.Cause wise mortality index shows that hypertensive disorders and infections are well managed. Women with heart disease are having high mortality.

The statistics from Kasthuriba hospital[4], Manipaul University, India during January 2011-December 2012(2 yrs) MNM ratio was 17.8 per 1000 live birth, maternal near miss mortality ratio is 5.6 and mortality index was $14.9 \%$. Haemorrhage was the leading cause of near miss $(44.2 \%)$, followed by hypertensive disorder $(23.6 \%)$ and sepsis $(16.3 \%)$. On comparing the incidence of near miss is more and their chance for survival is also high in our institution.

In a study reported from medical college of western Rajasthan[6], using Gellar's scoring system[11] India obstetric near miss rate is 4.18 per 1000 live births. Maternal death to near miss ratio was 1:2.07.major cause for maternal near miss is haemorrhage (58\%) and the second leading cause is hypertension $(17.8 \%)$. Incidence of near miss is low, may be because of using Gellar's scoring system.

A retrospective study of near miss obstetric event and maternal death in Sagamu, Nigeria [3] conducted between 2002-2004 (3yrs) showed near miss rate was 140.6 per 1000 live births. Near miss to maternal death ratio was 4.8:1.as like our institution the leading cause was hyper tension (31.4\%) followed by haemorrhage $(29 \%)$.

In Damascus maternity university hospital [7] in the year 2006-2007, maternal near miss rate reported was 32.9 per 1000 live birth. Hyper tension contributing to $52 \%$ and haemorrhage to $34 \%$. Mortality index being 1.7 which is low when compared with our institution (4.7).

\section{Results}

Analysis of the data revealed that the incidence of near miss is 20 fold higher than the maternal death. There is no significant difference in age distribution, parity and gestational age at the time of admission between near miss group and maternal death group. Hypertensive disorder is the leading cause for near miss. Medical 
complications like heart disease carries higher mortality index than obstetric complications. Perinatal and neonatal mortality is significantly higher in near miss group when compared with general population.

\section{Conclusion}

Maternal near miss is a potential indicator of maternal health. Notification and analysis of maternal near miss events will help in improving maternal health care system. It will also provide vital data for the policy makers to concentrate and strengthen the potential deficits so as to achieve safe mother hood.

\section{Reference}

[1] The WHO near-miss approach for maternal health; Evaluating the quality of care for severe pregnancy complications World Health Organization 2011()

[2] Maternal near miss review operational guidelines December 2014,Ministry of Health \& Family Welfare Government of India Maternal Health Division by Shri C. K. Mishra, AS \& MD (NHM), mohfw, Dr Rakesh Kumar, JS (RMNCH+A), mohfw, Dr Himanshu Bhushan, DC (I/c MH), MOHFW

[3] "Near-miss" obstetric events and maternal deaths in Sagamu, Nigeria: a retrospective study Olufemi T Oladapo, Adewale O Sule-Odu, Published: 01 November 2005; Reproductive Health 20052:9

[4] "Near Miss" Obstetric Events and Maternal Deaths in a Tertiary Care Hospital: An Audit Roopa PS, Shailja Verma, Kasturba Hospital, Manipal University, Manipal, India.

[5] Levels of Maternal Mortality Ratio (MMR) by Regions, 1997-2012

[6] Obstetric near miss morbidity and maternal mortality in a Tertiary Care Centre in Western Rajasthan, Priyanka Kalra, Chetan Prakash Kachhwaha Senior Resident, Department of Obstetrics and Gynaecology, Dr. S. N. Medical College, Jodhpur, Rajasthan, India. BRIEF RESEARCH ARTICLE Year : 2014 | Volume : 58 | Issue : 3 | Page : 199-201

[7] Obstetric near-miss and maternal mortality in maternity university hospital, Damascus, Syria: a retrospective studyyara Almerie, Muhammad Q AlmerieEmail author, BMC Pregnancy and Child birth 201010:65; Published: 19 October 2010

[8] WHO: International Classification of Diseases and Related Health Problems. 1992, Geneva, Switzerland: World Health Organization,

[9] Vandecruys H, Pattinson RC, Macdonald AP, Mantel GD: Severe acute maternal morbidity and mortality in the Pretoria Academic Complex: changing patterns over four years. Eur J Obstet Gynaecol Reprod Biol. 2002, 102 (1): 6-10. 10.1016/S03012115(01)00558-9.

[10] Waterstone M, Bewley S, Wolfe C: Incidence and predictors of severe obstetric morbidity: case-control study. BMJ. 2001, 322 (7294): 1089-1094. 10.1136/bmj.322.7294.1089.

[11] Geller SE, Rosenberg D, Cox S, Brown M, Simonson L, Kilpatrick S. A scoring system identified near-miss maternal morbidity during pregnancy. J Clin Epidemiol 2004; 57:716-20.

[12] Pattinson RC, Buchmann E, Mantel G, Schoon M, Rees H: Can enquiries into severe acute maternal morbidity act as a surrogate for maternal death enquiries? BJOG. 2003, 110: 889-893.View Article. 